\title{
On Benjamin-Feir instability and evolution of a nonlinear wave with finite-amplitude sidebands
}

\author{
L. Shemer \\ School of Mechanical Engineering, Tel-Aviv University, Tel-Aviv, Israel
}

Received: 9 August 2010 - Accepted: 7 September 2010 - Published: 30 November 2010

\begin{abstract}
In the past decade it became customary to relate the probability of appearance of extremely steep (the socalled freak, or rogue waves) to the value of the BenjaminFeir Index (BFI) that represents the ratio of wave nonlinearity to the spectral width. This ratio appears naturally in the cubic Schrödinger equation that describes evolution of unidirectional narrow-banded wave field. The notion of this index stems from the Benjamin-Feir linear stability analysis of Stokes wave. The application of BFI to evaluate the evolution of wave fields, with non-vanishing amplitudes of sideband disturbances, is investigated using the Zakharov equation as the theoretical model. The present analysis considers a 3-wave system for which the exact analytical solution of the model equations is available.
\end{abstract}

\section{Introduction}

Extreme (rogue, or freak) waves appear locally and spontaneously in the sea. Usually waves with heights exceeding twice the significant wave height are considered to belong to this category. Parameters of those waves, and in particular the frequency of their appearance, constitute an important design parameter in marine and off-shore engineering. There are numerous indications that under certain circumstances these waves may appear more frequently than expected for a random Gaussian sea. In recent years, numerous experimental studies aimed at experimental investigation of probability of appearance of extremely high waves were carried out under controlled conditions in large experimental facilities (Onorato et al., 2006, 2009; Shemer and Sergeeva, 2009; Waseda et al., 2009; Shemer et al., 2010 and additional references therein). Both unidirectional random wave fields and those with directional spreading were considered. Random

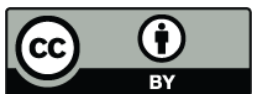

Correspondence to: L. Shemer (shemer@eng.tau.ac.il) waves with an initially narrow-banded unidirectional Gaussian spectrum were studied in Shemer and Sergeeva (2009). Three series of experiments were carried out for different values of nonlinearity determined by the characteristic wave amplitude. It was found that the frequency spectrum of the wave field undergoes significant variation in the course of the wave field evolution along the tank. The initially narrow spectrum becomes wider at the early stages of the evolution, up to the distance from the wavemaker ranging from about $70 \mathrm{~m}$ for the highest characteristic amplitude employed, to roughly twice that length for the weakest nonlinearity. After attaining maximum, the spectrum gets narrower, its width, however, remains larger than the initial one. The values of all the statistical wave parameters appear to be strongly related to the local spectral width. The deviations of various statistical parameters from the Gaussian statistics increase with the width of the spectrum so that the probability of extremely large waves is the highest when the local spectral width attains maximum. The probability of extremely steep waves also increases with the degree of nonlinearity.

The effect of the spectral width for a constant nonlinearity $\varepsilon$, defined as a product of the dominant wavenumber $k_{0}$ by the r.m.s. value of the surface elevation $\sigma=\left(\overline{\eta^{2}}\right)^{1 / 2}, \varepsilon=k_{0} \sigma$, was studied, e.g., in Onorato et al. (2006) and in Shemer et al. (2010) for a unidirectional wave field, and in Onorato et al. (2009) in a large basin for a constant frequency spectrum but with varying directional spreading. These studies demonstrated that as either the frequency range or the directional spreading widen, the probability of appearance of extremely steep waves decreases.

To describe the evolution of random nonlinear wave fields and following Alber (1978) and Onorato et al. (2001), Janssen (2003) introduced the Benjamin-Feir index, $\mathrm{BFI}=2^{1 / 2} \varepsilon /\left(\Delta \omega / \omega_{0}\right)$, as a ratio of nonlinearity $\varepsilon$ to the relative spectral width $\Delta \omega / \omega_{0}, \omega_{0}$ being the carrier wave frequency and $\Delta \omega$ the width of the frequency spectrum; $\Delta \omega / \omega_{0}$ can be seen as a measure of wave dispersion.

Published by Copernicus Publications on behalf of the European Geosciences Union. 
Janssen stressed that BFI can be expected to fully characterise the evolution of a unidirectional wave field only within the framework of the cubic Schrödinger equation (CSE) valid for a very narrow spectrum, whereas for wider spectra this is not the case. Nevertheless, quantitative estimates pertaining to the character of random wave fields' evolution that are based on BFI are quite common. For example, Onorato et al. (2006) use BFI to analyse the experiments carried out for a relatively wide JONSWAP spectrum, while Onorato et al. (2009) even invoke BFI in their experimental study of 2-D random waves.

While there is a general consensus that BFI can be useful as a rough indicator characterising the random field evolution pattern, application of this index as a quantitative predictive tool raises some questions. One commonly acknowledged problem with the BFI is a non-unique way in which both the nonlinearity $\varepsilon$ and the spectral width $\Delta \omega / \omega_{0}$ are defined. For example, the value of $\omega_{0}$ is often defined as corresponding to the spectral peak. A more robust definition of the dominant wave frequency (and, thus, via the dispersion relation, of the dominant wavenumber $k_{0}$ ) in a random wave field would invoke spectral momentum based of the free waves spectra (see, e.g., Shemer et al., 2010). Similarly, the dimensionless spectral width $\Delta \omega / \omega_{0}$ is sometimes defined according to the energy level that corresponds to half of the spectral peak value, whereas again, the definition based on spectral momenta seems more appropriate. Alternative possible definitions of those parameters can result in quantitatively different values of BFI for identical wave spectra, especially for wider and asymmetrically shaped spectra like JONSWAP.

An additional problem related to BFI is addressed here. By its very nature, this index is closely related to the BenjaminFeir instability of the Stokes waves, and the coefficient preceding the ratio of nonlinearity to dispersion in the definition of BFI reflects either the most unstable sideband disturbance according to CSE, or the limit of the sideband linear stability in a slightly different definition of BFI. Moreover, it is quite common to refer to nonlinear evolution of the wave field as stemming from the Benjamin-Feir modulational instability.

The Benjamin-Feir (BF) instability is a result of linear analysis of a system that consists of a carrier wave with amplitude $a_{0}$ and wave vector $\boldsymbol{k}_{0}$, and two infinitesimal sideband disturbances with wave vectors $\boldsymbol{k}_{1}$ and $\boldsymbol{k}_{2}$ that satisfy the condition

$2 \boldsymbol{k}_{0}=\boldsymbol{k}_{1}+\boldsymbol{k}_{2}$.

The linearized (with respect to the sideband disturbances amplitudes) governing equations are used to carry out the stability analysis that delineates the boundaries of Stokes wave instability domain and allows determining the most unstable modes. Random wave fields have spectral "tails" extending well beyond the wavenumber domain around the dominant wave determined by the spectral width. The amplitude of those spectral components, while small relative to that of the dominant wave, is finite. It is, thus, instructive to consider nonlinear evolution of a 3-wave system consisting of a carrier wave with a given steepness, and two side disturbances with initially much smaller but still finite amplitudes. The three-wave system defined by (1) can be seen as a basic "molecule" in the evolution pattern representing one of the numerous possible nonlinear interactions in a wave field with a given spectrum. The advantage of such a system is that it allows an exact analytic solution of the wave evolution problem based on the Zakharov (1968) equation (Shemer and Stiassnie, 1985; Stiassnie and Shemer, 2005). The availability of the analytic solution enables the parametric study of the 3-wave system that can shed light on behaviour of much more complicated random wave fields.

\section{Theoretical background}

The nonlinear evolution of each of the waves due to quartet interactions at the $3 \mathrm{rd}$ order in the nonlinearity parameter $\varepsilon$ is described by the Zakharov equation

$$
\begin{aligned}
& i \frac{\partial B_{j}}{\partial t}=\iint_{-\infty}^{\infty} \int_{j, l, m, n} B_{l}^{*} B_{m} B_{n} \delta \\
& \left(\boldsymbol{k}_{j}+\boldsymbol{k}_{l}-\boldsymbol{k}_{m}-\boldsymbol{k}_{n}\right) e^{i \Delta_{j, l, m, n} t} d \boldsymbol{k}_{l} d \boldsymbol{k}_{m} d \boldsymbol{k}_{n}
\end{aligned}
$$

where the interacting quartets are defined by the $\delta$-function and the frequency detuning

$\Delta_{j, l, m, n}=\omega_{j}+\omega_{l}-\omega_{m}-\omega_{n}$

The interaction coefficients $V_{j, l, m, n}=V\left(\boldsymbol{k}_{j}, \boldsymbol{k}_{l}, \boldsymbol{k}_{m}, \boldsymbol{k}_{n}\right)$ are given in Krasitskii (1994). The free-surface elevation is related to the generalized amplitude $B(\boldsymbol{k}, t)$ by

$$
\eta=\frac{1}{2 \pi} \int_{-\infty}^{\infty}\left(\frac{|k|}{2 \omega}\right)^{1 / 2}\left\{B e^{i(\boldsymbol{k} \times x-\omega t)}+c . c\right\} d \boldsymbol{k},
$$

where the frequency $\omega$ is related to wave vector $\boldsymbol{k}$ by the deep-water linear dispersion relation

$\omega^{2}=g|\boldsymbol{k}|$

Following Stiassnie and Shemer (1984) and Shemer and Stiassnie (1985), consider an interaction wave quartet consisting of a carrier wave with the normalized wave vector $\boldsymbol{k}_{0}=(1,0)$ that is taken twice, and a pair of sideband disturbances $\boldsymbol{k}_{1,2}=(1 \pm p, \pm q)$, so that (1) is satisfied. The deviation from unity of the wavenumber is denoted as $p_{\mathrm{m}}$ for the most unstable pair of disturbances and $p_{\ell}$ for the limit of the linear instability. The linear instability analysis based on the CSE yields $p_{\ell}=2^{1 / 2} p_{\mathrm{m}}=2^{3 / 2} \varepsilon$, where the nonlinearity $\varepsilon=k_{0} a_{0}$ represents the carrier wave steepness. The 
Janssen's (2003) comment regarding the limits of applicability of BFI is based, in part, on the fact that the linear relation between $p_{\ell}$ (or $p_{\mathrm{m}}$ ) and $\varepsilon$ breaks down when the stability analysis is performed using either the linearized $\mathrm{Za}$ kharov equation (Crawford et al., 1981; Stiassnie and Shemer, 1984), or full nonlinear equations (McLean et al., 1981). The dependence of $p_{\mathrm{m}}$ and $p_{\ell}$ on the carrier wave steepness $\varepsilon$ is plotted in Fig. 1 according to calculations based on the cubic Schrödinger and Zakharov equations. As demonstrated by Stiassnie and Shemer (1984), the linear stability results based on the Zakharov equation do not differ notably from those obtained by McLean et al. It is obvious from Fig. 1 that the two models yield identical results only for vanishingly small values of $\varepsilon$; for steeper carrier waves, the CSE-derived values of $p_{\ell}$ and $p_{\mathrm{m}}$ are significantly higher than those based on the more accurate Zakharov model.

The main goal of this study is to analyse nonlinear interactions of a carrier wave with sidebands of initially finite amplitude. For a 3-wave system considered here, the integrodifferential equation (2) degenerates into a system of three first-order nonlinear ordinary differential equations:

$i \frac{d B_{0}}{d t}=\left(\Omega_{0}-\omega_{0}\right) B_{0}+2 V_{0,0,1,2} e^{i \Delta \omega t} B_{0}^{*} B_{1} B_{2}$

$i \frac{d B_{1}}{d t}=\left(\Omega_{1}-\omega_{1}\right) B_{1}+2 V_{1,2,0,0} e^{-i \Delta \omega t} B_{2}^{*} B_{0}^{2}$

$i \frac{d B_{2}}{d t}=\left(\Omega_{2}-\omega_{2}\right) B_{2}+2 V_{2,1,0,0} e^{-i \Delta \omega t} B_{1}^{*} B_{0}^{2}$

where the frequency detuning

$\Delta \omega=\Delta_{0,0,1,2}=2 \omega_{0}-\omega_{1}-\omega_{2}$

and the so-called "Stokes-corrected" frequencies are:

$\Omega_{0}=\omega_{0}+V_{0,0,0,0}\left|B_{0}\right|^{2}+2 V_{0,1,0,1}\left|B_{1}\right|^{2}+2 V_{0,2,0,2}\left|B_{2}\right|^{2}$

$\Omega_{1}=\omega_{1}+2 V_{1,0,1,0}\left|B_{1}\right|^{2}+V_{1,1,1,1}\left|B_{1}\right|^{2}+2 V_{1,2,1,2}\left|B_{2}\right|^{2}$

$\Omega_{2}=\omega_{2}+2 V_{2,0,2,0}\left|B_{0}\right|^{2}+2 V_{2,1,2,1}\left|B_{1}\right|^{2}+V_{2,2,2,2}\left|B_{2}\right|^{2}$

Following Stiassnie and Shemer (2005), after some manipulations one obtains from (6)

$$
\begin{aligned}
& \frac{d}{d t}\left|B_{0}\right|^{2}=-\frac{d}{d t}\left|B_{1}\right|^{2}=-\frac{d}{d t}\left|B_{2}\right|^{2} \\
& =4 V_{0,0,1,2} \operatorname{Im}\left\{\left(B_{0}^{*}\right)^{2} B_{1} B_{2} e^{i \Delta \omega t}\right\}
\end{aligned}
$$

The complex amplitudes of all three waves at all times, thus, depend on a single auxiliary real function $Z(t)$ defined by

$\frac{d Z}{d t}=\operatorname{Im}\left\{\left(B_{0}^{*}\right)^{2} B_{1} B_{2} e^{i \Delta \omega t}\right\}, \quad Z(0)=0$

It follows from (9) and (10) that

$\left|B_{0}\right|^{2}-\left|\beta_{0}\right|^{2}=-\left|B_{1}\right|^{2}+\left|\beta_{1}\right|^{2}=-\left|B_{2}\right|^{2}+\left|\beta_{2}\right|^{2}=4 V_{0,0,1,2} Z$

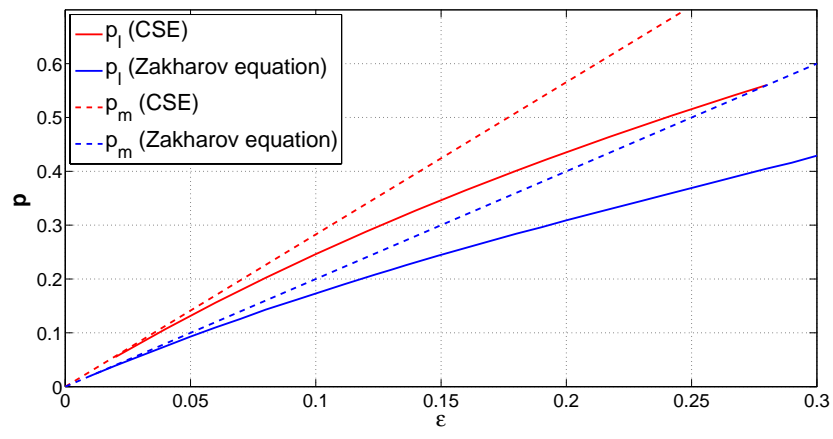

Fig. 1. The dependence of values of $p$ corresponding to the limit of linear instability $\left(p_{\ell}\right)$ and to the linearly most unstable disturbance $\left(p_{\mathrm{m}}\right)$ on the carrier wave steepness according to the NLS and Zakharov equations.

where the initial complex amplitudes $\beta_{j}=B_{j}(0), j=0,1,2$. From (6) and (10) one can show that

$\frac{d}{d t} \operatorname{Re}\left\{\left(B_{0}^{*}\right)^{2} B_{1} B_{2} e^{i \Delta \omega t}\right\}=-\Omega \frac{d Z}{d t}$

where

$\Omega=2 \Omega_{0}-\Omega_{1}-\Omega_{2}$.

In view of (8) and (11), $\Omega$ is a linear function of $Z$ that can be presented as

$\Omega=\Omega_{a}+\Omega_{b} Z$.

Integrating (12) from $t=0$ gives

$\operatorname{Re}\left\{\left(B_{0}^{*}\right)^{2} B_{1} B_{2} e^{i \Delta \omega t}\right\}=\operatorname{Re}\left\{\left(\beta_{0}^{*}\right)^{2} \beta_{1} \beta_{2}\right\}-\int_{0}^{Z} \Omega d Z$

Using (10) and (15) yields

$\left(\frac{d Z}{d t}\right)^{2}=\left|B_{0}\right|^{4}\left|B_{1}\right|^{2}\left|B_{2}\right|^{2}$

$-\left[\operatorname{Re}\left\{\left(\beta_{0}^{*}\right)^{2} \beta_{1} \beta_{2}\right\}-\Omega_{0} Z-\frac{\Omega_{1}}{2} Z^{2}\right]^{2}=P_{4}(Z)$

The coefficients of the 4 th order polynomial $P_{4}(Z)$ are fully determined by the initial conditions. It follows from (16) that

$t=\int_{0}^{Z} d Z / \sqrt{P_{4}(Z)}$

It is possible to express the integral (17) in terms elliptic functions (Byrd and Friedman, 1971). For wave parameters considered in this study, the coefficient of the leading term $Z^{4}$ in the polynomial is positive in most cases, all roots of $P_{4}(Z)$ are real and can be arranged so that $Z_{4}>Z_{3}>$ $0>Z_{2}>Z_{1}$. The following derivation is carried out for this case, nevertheless, using appropriate expressions in Byrd and 
Friedman it can easily be modified to cases when the roots of the polynomial $P_{4}(Z)$ are arranged differently. For the case considered, the value of $Z$ can vary between $Z_{2}$ and $Z_{3}$, so that the solution (17) is periodic with the period given by

$T=2 \int_{Z_{2}}^{Z_{3}} d Z / \sqrt{P_{4}(Z)}$

Shemer and Stiassnie (1985) and Stiassnie and Shemer (2005) presented the following explicit expression for (17):

$Z=\frac{Z_{4}\left(Z_{3}-Z_{2}\right) s n^{2} u-Z_{3}\left(Z_{4}-Z_{2}\right)}{\left(Z_{3}-Z_{2}\right) s n^{2} u-\left(Z_{4}-Z_{2}\right)}$

where $s n$ is the Jacobi elliptic function of argument

$u=s n^{-1}(\delta, \kappa)-a_{o}^{1 / 2} t / \gamma$

and modulus

$\kappa=\sqrt{\frac{\left(Z_{3}-Z_{2}\right)\left(Z_{4}-Z_{1}\right)}{\left(Z_{4}-Z_{2}\right)\left(Z_{3}-Z_{1}\right)}}$

In (20),

$\gamma=\frac{2}{\sqrt{\left(Z_{4}-Z_{2}\right)\left(Z_{3}-Z_{1}\right)}}, \quad \delta=\sqrt{\frac{Z_{3}\left(Z_{4}-Z_{2}\right)}{Z_{4}\left(Z_{3}-Z_{2}\right)}}$

The modulation period (18) can be rewritten as

$T=\frac{2 \gamma}{a_{o}^{1 / 2}} K(\kappa)$,

where $K$ is the complete elliptic integral of the first kind. Note, that wave complex amplitudes $B$ depend on $Z$ and are, therefore, periodic; it also follows from (9) and (11) that the solution does not depend on the individual initial phases of the participating harmonics, but rather on a single initial phase given by

$\theta=2 \arg \beta_{0}-\arg \beta_{1}-\arg \beta_{2}$.

Thus, the solution, in terms of the Jacobi elliptic functions, allows computing the variation of the amplitude of each of the three wave components as a function of their initial amplitudes and of a single phase combination $\theta$.

\section{Results}

In all cases considered, the initial sideband amplitudes are defined relative to that of the carrier wave, so that $a_{1,2}=$ $c_{1,2} a_{0}$. The coefficients $c_{1,2}$ mostly do not exceed 0.4 , so that the power of sidebands remains well below $20 \%$. Therefore, these sidebands are beyond the energy-containing part of the spectrum and can be seen as belonging to spectral tails that practically do not contribute to the effective width of the

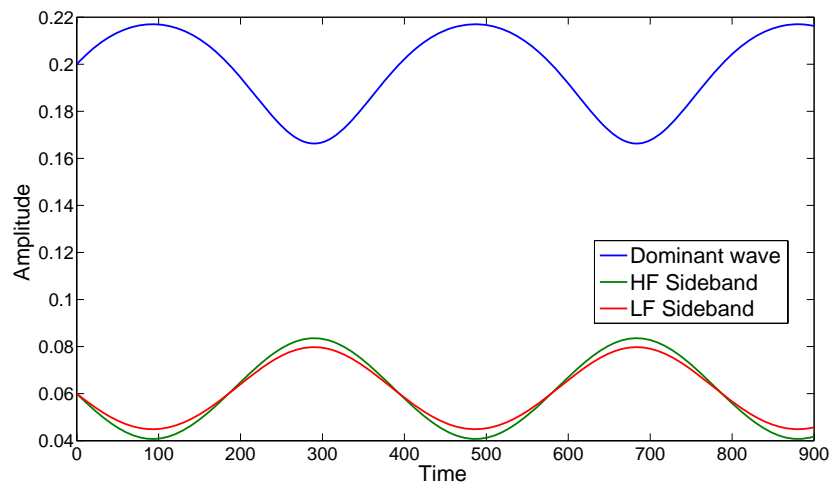

Fig. 2. Nonlinear evolution of a 3-wave system: $p=0.2 ; q=0$; $\theta=0.7 \pi ; \varepsilon=0.2 ; c_{1}=c_{2}=0.3$.

spectrum in any conceivable definition of this parameter. In deep water the most unstable infinitesimal disturbances propagate in the direction of the carrier wave (McLean et al., 1981; Stiassnie and Shemer, 1984), so that the vectors $\boldsymbol{k}_{1}$ and $\boldsymbol{k}_{2}$ are parallel to the carrier wave vector $\boldsymbol{k}_{0}$ and $q=0$. Unidirectional wave systems are, thus, considered first.

The results of the previous section indicate that a threewave system undergoes periodic Fermi-Pasta Ulam recurrence for an arbitrary value of the sideband wavenumber deviation $p$. A typical example of the temporal evolution of a unidirectional 3-wave system is presented in Fig. 2 for the carrier wave steepness $\varepsilon=0.2$ and identical initial relative amplitudes of sidebands $c_{1}=c_{2}=0.2$. The sidebands selected in this example belong to the BF instability domain, see Fig. 1. The dimensionless modulation period $T$ that characterises the exchange of energy between the carrier wave and both sidebands can be computed using (23). For the conditions of Fig. 2, the dimensionless $T=393.8$, or about 62 periods of the carrier wave. This is in agreement with the so called "dynamic" temporal evolution scale of the order of $\varepsilon^{-2}$ appropriate for 4-wave nonlinear interactions.

As follows from (11), the amplitude of periodic modulation of each one of the 3 components is determined by a single real variable $Z$; the range of variation in $Z, \Delta Z$, for given initial conditions defines the extent of modulation and can, thus, be seen as a measure of the intensity of nonlinear interactions at a slow time scale. The domain of variation $\Delta Z$ is determined solely by the values of the roots of the polynomial $P_{4}(Z)$.

The effect of the initial amplitude of sidebands is investigated in Fig. 3 for a unidirectional wave system with the carrier wave amplitude $\varepsilon=0.2$, as a function of the sideband wavenumber determined by the value of $p$. In Fig. 3a, both sidebands are assumed to have identical initial amplitude, whereas in Fig. $3 \mathrm{~b}$ and c, the initial amplitudes of either the low frequency sideband with $\boldsymbol{k}_{3}=(1-p, 0)$, or the high frequency sideband with $\boldsymbol{k}_{2}=(1+p, 0)$, respectively, are set as zero. In those cases, the initial amplitude of the initially 
non-zero sideband is increased by a factor of $2^{1 / 2}$ compared to the corresponding values in Fig. 3b, to keep the total power of all 3 components identical in Fig. 3. The range of variation of $p$ extends in Fig. 3 well beyond the linear stability limits computed using either the NLS or the Zakharov equations, as plotted in Fig. 1.

For the case of identical sideband amplitudes considered in Fig. 3a, the intensity of nonlinear interactions characterised by $\Delta Z$ increases monotonically with the amplitude of sidebands. In the whole range of values of $p$ considered, the values of $\Delta Z$ vary continuously with $p$ and do not vanish, although they decrease significantly for wider-spaced sidebands. For higher initial sideband amplitudes, significant nonlinear interactions exist even well beyond the BF stability domain. The linear stability limit (corresponding to $p \approx 0.44$ for the BF stability analysis based on the Zakharov equation) does not play any particular role. Moreover, the modulation depth does not attain maximum for the most unstable disturbance. The exact value of $p$ where the intensity of nonlinear interactions attains maximum depends on the initial amplitude of the sidebands.

The evolution of an asymmetric wave system in which only one sideband exists initially does not differ qualitatively from that observed for the case of initially symmetric sideband disturbances. However, notable quantitative differences exist. For sideband wavenumbers adjacent to that of the carrier wave, the modulation intensity in Fig. $3 \mathrm{~b}$ and $\mathrm{c}$ is smaller by a factor close to 2 as compared to that in Fig. 3a. The values of $\Delta Z$ then decay faster with increase in $p$, although decrease in the amplitude of modulations is less steep in Fig. $3 \mathrm{c}$ where the low-frequency sideband initially has non-zero amplitude, as compared to Fig. 3b. In Fig. $3 b$ and $c$ the most intensive nonlinear interactions are obtained for sidebands that are not too far from the carrier wave, rather than for the values of $p$ corresponding to the most unstable disturbances in the linear $\mathrm{BF}$ analysis.

The results of Fig. 3 can be supported by findings of Shemer and Chamesse (2000) who studied experimentally the BF instability of gravity-capillary waves on the basis of the Zakharov equation. In the experiments a carrier wave was generated by a plane wavemaker, while one of the sidebands (with a frequency either higher or lower than that of the carrier) was excited with a small but finite amplitude by an independent auxiliary conical wavemaker; its frequency was varied in a range exceeding the instability domain. The appearance in the measured spectrum of the sideband that has not been excited directly was interpreted as a manifestation of the BF instability. In the measured spectra, however, this sideband was often visible even when the auxiliary wavemaker was operated at frequencies outside of the instability domain. The results of Fig. 3 are in general agreement with those observations.

The examples presented in Figs. 2 and 3 were computed for an arbitrarily selected initial phase angle combination $\theta=0.7 \pi$. The effect of $\theta$ on the intensity of nonlinear in-

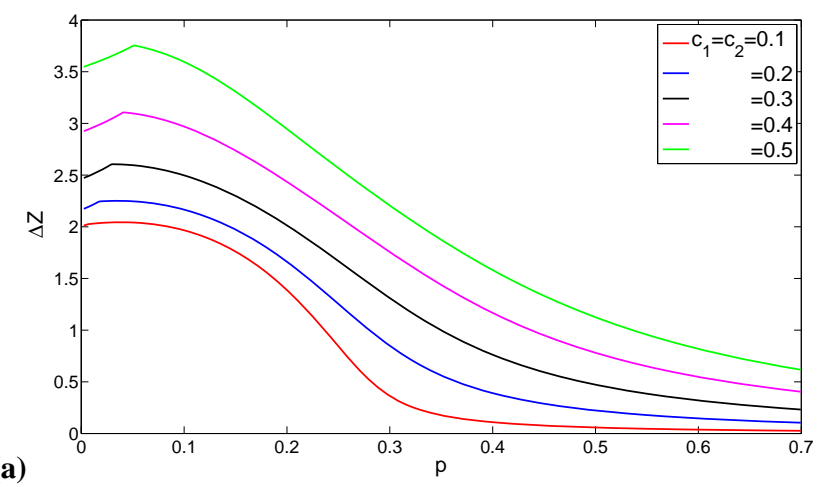

(a)

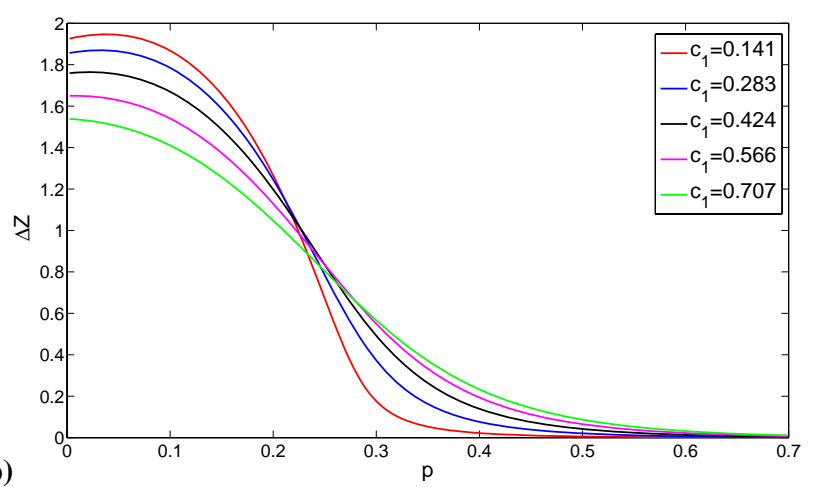

(b)

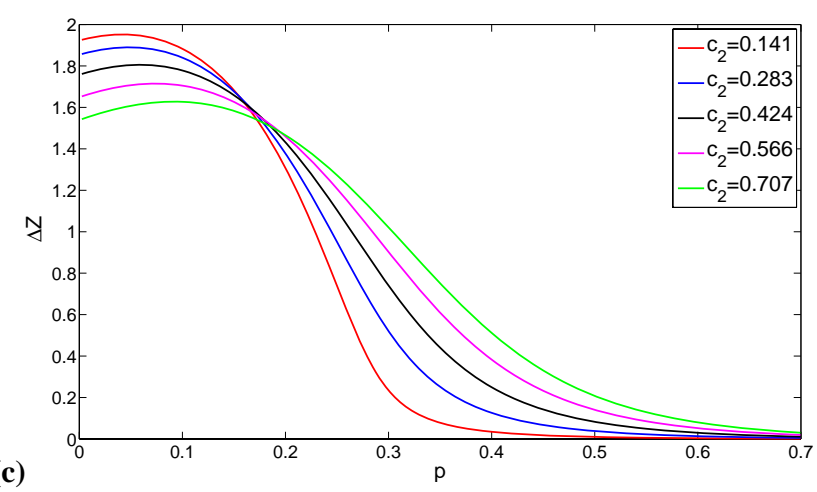

Fig. 3. Effect of the relative initial sideband amplitude on the modulation depth for $\varepsilon=0.2$ and $\theta=0.7 \pi$ : (a) $c_{1}=c_{2}$; (b) initial low frequency sideband amplitude $c_{3}=0$; (c) initial high frequency sideband amplitude $c_{2}=0$.

teractions is studied in Fig. 4 for the carrier wave amplitude $\varepsilon=0.2$ and a set of identical initial sideband amplitudes. In Fig. $4 \mathrm{a}$, the sidebands that are relatively close to the carrier wave, $p=0.2$, are considered, while in Fig. $4 \mathrm{~b} p=0.45$, beyond the limits of the BF linear instability. In Fig. $4 a$, the phase dependence of $\Delta Z$ increases strongly with the initial sideband amplitude. For phase combination angles sufficiently close to zero, the modulation intensity may become even stronger for initially smaller sideband amplitudes. For widely-spaced sidebands in Fig. $4 \mathrm{~b}$, the phase dependence of $\Delta Z$ weakens notably; the values of $\Delta Z$ increase with the initial amplitude of sidebands for all initial phases $\theta$. Note that 
(a)

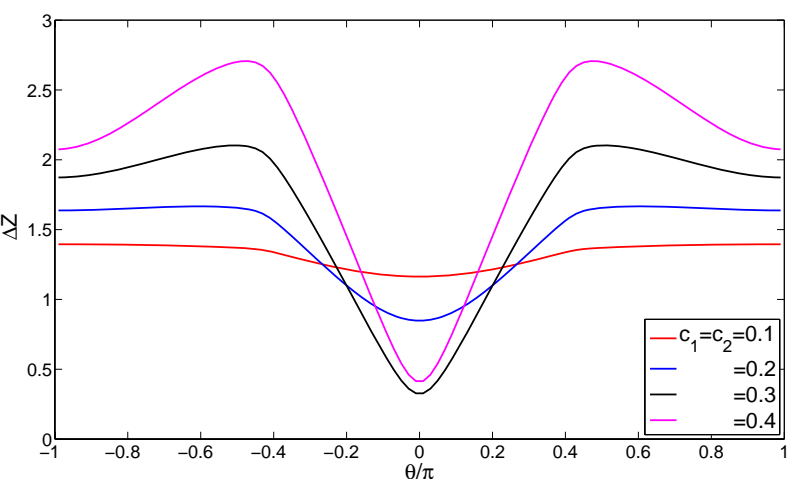

(b)

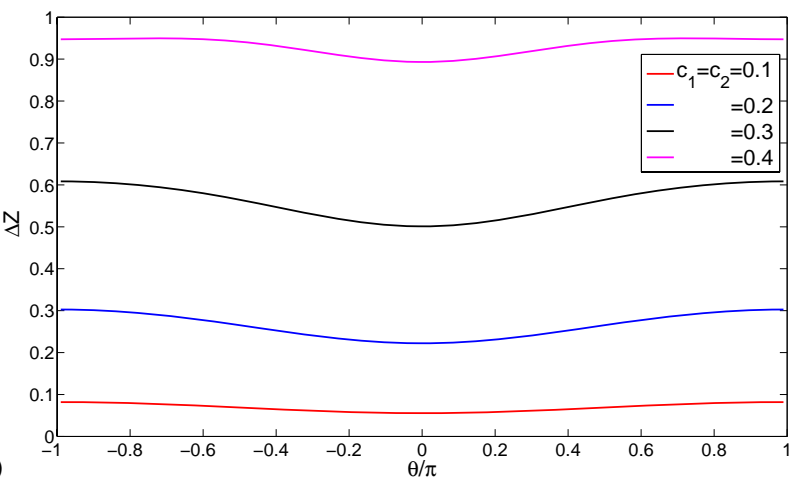

Fig. 4. Modulation intensity for the carrier wave steepness $\varepsilon=0.2$ as a function of the initial phase angle $\theta$ for: (a) $p=0.2$; (b) $p=0.45$.

for $c_{1}=c_{2}=0.4$, the modulation amplitude in the vicinity of $\theta=0$ is higher for $p=0.45$ in Fig. $4 \mathrm{~b}$ as compared to that for $p=0.2$ in Fig. 4 a.

Finally, the effect of directional spreading is examined in Fig. 5 for $\theta=0.7 \pi$, other conditions being as in Fig. 4. Note that according to the exact linear stability analysis of finiteamplitude waves by McLean et al. (1981), the instability limits are $0 \leq q \leq 0.125$ for $p=0.2$, and $0 \leq q \leq 0.25$ for $p=0.45$. As evident from Fig. 4a, for sidebands with finite initial amplitude the nonlinear interactions are quite strong well beyond those limits. Even more unexpected may be the results of Fig. $4 \mathrm{~b}$ that indicate that the most significant nonlinear interactions occur for wave vectors that correspond to linearly stable disturbances. These interactions result in sideband amplitudes that, for certain phases of the Fermi-PastaUlam recurrence process, can exceed that of the carrier, even for initially relatively small values of $c_{1,2}$.

\section{Conclusions}

The notion of BFI was coined considering a 3-wave system consisting of a carrier and initially vanishingly small sideband disturbances. The 3-wave approach intrinsic to the BFI concept is expanded here to include sidebands that, while sufficiently small not to affect notably the spectral width, have nevertheless finite initial amplitudes. The Zakharov (a)

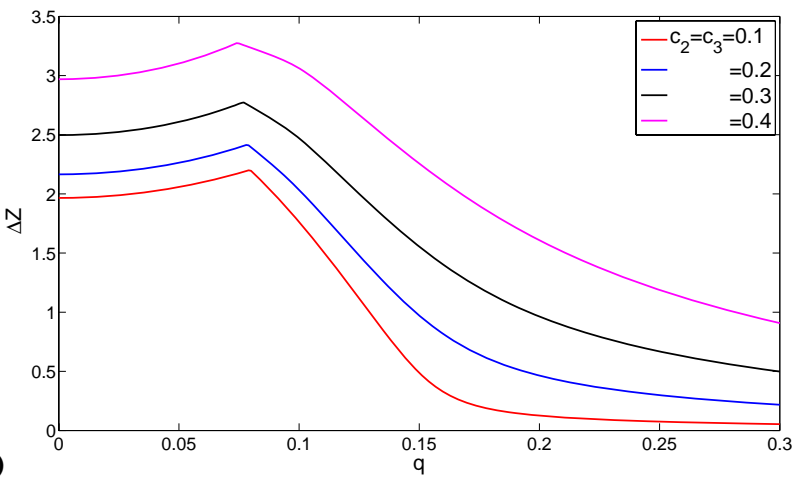

(b)

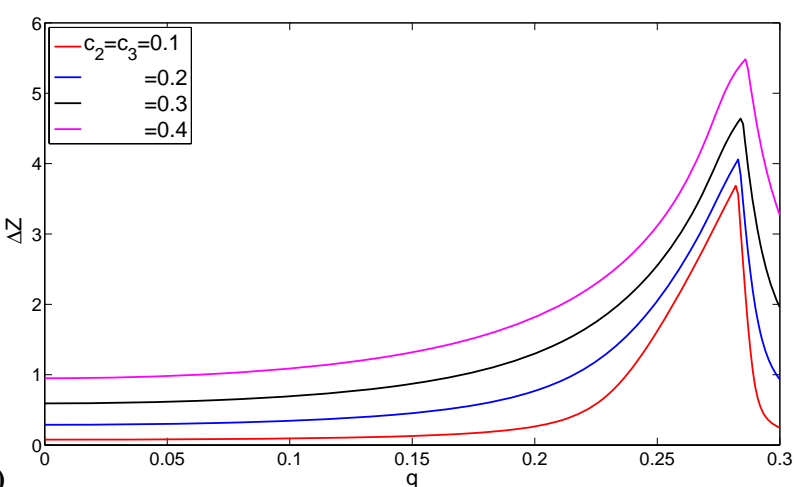

Fig. 5. Modulation intensity for the carrier wave steepness $\varepsilon=0.2$ and the initial phase angle $\theta=0.7 \pi$ as a function of $q$ for: (a) $p=0.2$; (b) $p=0.45$.

equation is used as the theoretical model. Contrary to envelope equations (the CSE and its modifications), it does not pose any restrictions on the spectral width. Advantage is taken of the availability of an exact solution of the resulting system of equations in terms of Jacoby elliptic functions. The analytical solution demonstrates that the 3-wave system undergoes periodic Fermi-Pasta-Ulam recurrence. The intensity of nonlinear interactions can be characterised by a single real parameter $\Delta Z$.

The present parametric study suggests that conclusions based on the linear stability analysis in general, and in particular when the CSE is used, are largely irrelevant for a 3-wave system in which initial sideband amplitudes are small but finite. The Benjamin-Feir analysis that considers the stability of a finite amplitude Stokes wave to infinitesimal sideband disturbances, therefore, cannot be seen as meaningful representation of the evolution of a nonlinear wave field. The most significant nonlinear interactions are not necessarily related to the linearly most unstable disturbances. The domains of the BF instability determined either by approximate models, such as narrow-banded CSE and the Zakharov equation, or by an application of fully nonlinear water-wave equations, cannot serve as indicators of the intensity of nonlinear interactions of a carrier wave with finite amplitude sidebands. 
The present results indicate that for a unidirectional wave field and initial disturbances with non-vanishing amplitudes, the values of sidebands' wavenumber deviation from that of the carrier $p$ and the carrier wave steepness $\varepsilon$ do not uniquely define the nonlinear evolution pattern of the whole wave system. This pattern appears to be crucially dependent also on the initial sideband amplitudes and often on their phases as well.

When sideband disturbances are allowed to propagate in directions different from that of the carrier wave, as is the case in laboratory studies in wave basins, or in field experiments, the spectral width, calculated in most experimental investigations from the measured frequency spectrum, becomes even less dominant in governing the nonlinear evolution process, which becomes crucially dependent not just on the sidebands' initial amplitude but on their directions as well.

The results of the present exercise with a simple deterministic nonlinear wave system can be useful in analysing much more complicated random wave fields. It should be kept in mind, however, that statistics of random water waves in laboratory and in sea is determined by a large number of interacting triads and quartets, each having random combinations of phases. Thus, extreme care should be exercised in the application of the present findings to a wider context of statistics of random nonlinear wave fields.

Acknowledgements. This work was supported by a grant \# 1194/07 from the Israel Science Foundation.

Edited by: E. Pelinovsky

Reviewed by: A. Babanin and another anonymous referee

\section{References}

Alber, I. E.: The effects of randomness on the stability of two dimensional surface wave trains, Proc. R. Soc. Lon. Ser.-A, 636, 525-546, 1978.

Byrd, P. F. and Friedman, M. P.: Handbook of elliptic integrals for engineers and scientists, Springer-Verlag, 358 pp., 1971.

Crawford, D. R., Lake, B. M., Saffman, P. G., and Yuen, H. C.: Stability of weakly nonlinear deep-water waves in two and three dimensions, J. Fluid Mech., 105, 177-192, 1981.
Janssen, P. A. E. M.: Nonlinear four-wave interactions and freak waves, J. Phys. Ocean., 33, 863-883, 2003.

Krasitskii, V. P.: On the reduced equations in the Hamiltonian theory of weakly nonlinear surface waves, J. Fluid Mech., 272, 120, 1994.

McLean, J. W., Ma, Y. C., Martin, D. U., Saffman, P. G., and Yuen, H. C.: Three-dimensional instability of finite-amplitude water waves, Phys. Rev. Letl., 46, 817-820, 1981.

Onorato, M., Cavalieri, L., Fouques, S., Gramstad, O., Jansses, P. A. E. M., Monbaliu, J., Osborne, A. R., Pakozdi, C., Serio, M., Stansberg, C. T., Toffoli, A., and Trulsen, K.: Statistical properties of mechanically generated surface gravity waves: a laboratory experiment in a three-dimensional wave basin, J. Fluid Mech., 627, 235-257, 2009.

Onorato, M., Osborne, A. R., Serio, M., and Bertone, S.: Freak wave in random oceanic sea states, Phys. Rev. Lett., 86, 58315834, 2001.

Onorato, M., Osborne, A. R., Serio, M., Cavalieri, L., Brandini, C., and Stansberg, C. T.: Extreme waves, modulational instability and second order theory:wave flume experiments on irregular waves, Eur. J. Mech. B-Fluid., 25, 586-601, 2006.

Shemer, L. and Chamesse, M.: Experiments on nonlinear gravitycapillary waves, J. Fluid Mech., 380, 205-232, 1999.

Shemer, L. and Sergeeva, A.: An experimental study of spatial evolution of statistical parameters in a unidirectional narrowbanded random wavefield, J. Geophys. Res., 114, C01015, doi:10.1029/2008JC005077, 2009.

Shemer, L. and Stiassnie, M.: Initial instability and long-time evolution of Stokes waves, in: The Ocean Surface: Wave Breaking, Turbulent Mixing and Radio Probing, edited by: Toba, Y. and Mitsuyasu, H., D. Reidel, Dordrecht, Holland, 51-57, 1985.

Shemer, L., Sergeeva, A., and Liberzon, D.: Effect of the initial spectrum on spatial evolution of the statistics of unidirectional nonlinear random waves, J. Geophys. Res., in press, 2010.

Stiassnie, M. and Shemer, L.: On modification of Zakharov equation for surface gravity waves, J. Fluid Mech., 143, 47-67, 1984.

Stiassnie, M. and Shemer, L.: On the interaction of four waterwaves, Wave Motion, 41, 307-328, 2005.

Waseda, T., Kinishita, T., and Tamura, H.: Evolution of a random directional wave and freak wave occurrence, J. Phys. Oceanogr., 39, 621-639, 2009.

Zakharov, V.: Stability of periodic waves of finite amplitude on the surface of a deep fluid, J. Appl. Mech. Tech. Phy., 9, 190-194, 1968. 